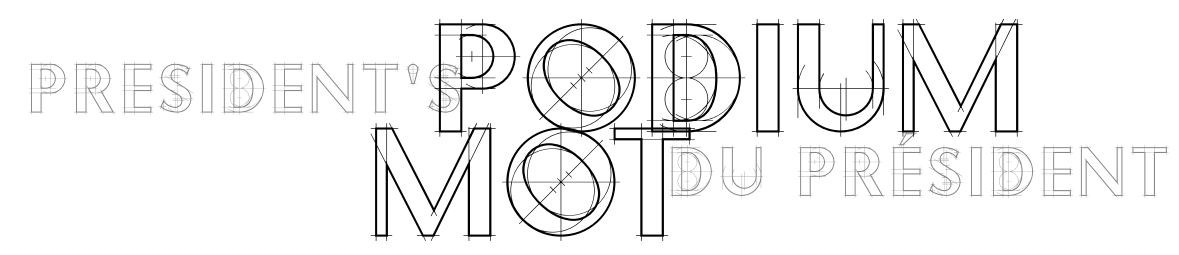

\title{
Turning the Page
}

\section{Tourner la page}

A

s we close the book on 2007 I believe we are poised to 'make 2008 great!' 1 am pleased to report that by virtually any measure, financially, operationally or strategically that CAO had a successful 2007 and that we have already begun building on that momentum in 2008 . Before turning the page let's review some 2007 highlights:

- Realizing the most financially successful Biennial Congress in CAO's history. Thank you Saskatoon and all attendees!

- Celebrating the 40th Anniversary of the School of Optometry's move to Waterloo.

- Another successful Eye Health Month. National cohesiveness on this project is absolutely amazing and the envy of many other professional organizations.

- Implementing and monitoring the CAO strategic plan for improved government relations, elevating optometry's standard of care, improving internal and external communications and updating CAO office infrastructure. This master plan also includes incorporating the OLF recommendations for improved communications, education and collaboration to create increased recognition for optometry.

- Preliminary planning for a major study to determine a recommended frequency of eye examinations for the Canadian public.
- Renovations to the CAO office are now complete, and within budget.

- Hiring a new employee, primarily responsible for monitoring our website - our future media for information, education and communications.

- Providing funding for a U of W School of Optometry Forum discussing projections for the future of optometric education.

- Initiating a program of free CAO membership (via application) for optometric students enrolled in all years in all North American Schools.

- Responding to the Competition Bureau report.

- Corresponding to provincial Health Ministers in $\mathrm{BC}, \mathrm{MB}$ and PEI concerning the compelling need to implement TPA legislation.

- Adopting the theme of 'Communication is the Key' - a recognition that continued public and intra professional education and promotion of optometric services will open many doors for our future success.

2007 was a good year, and we're on a roll.

Fast-forward to Jan 2008 and a record attendance at our 8th annual Optometric Leader's Forum (OLF). This is our early New Year's national meeting opportunity

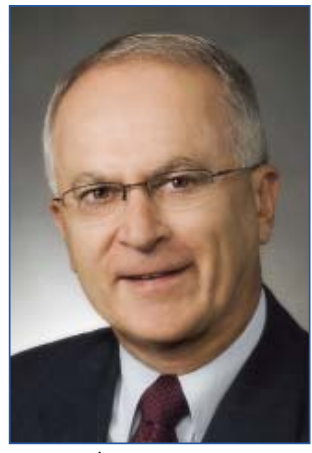

Len Koltun, OD President CAO / président de I'ACO

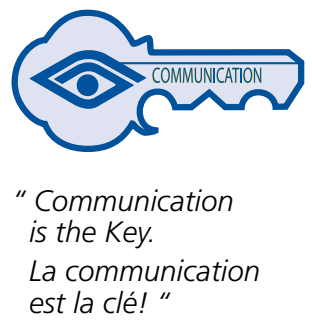




\section{PRESIDENT'S PODIUM MOT DU PRÉSIDENT}

to revise, rededicate and kick-start commitments to our goals for 2008.

The 2008 OLF experience was an excellent open and free exchange of ideas providing a spiritual rejuvenation, which really must be experienced to be appreciated. We are now better able to see the forest and the trees and I believe we will continue to be successful by creating and capturing new opportunities then capitalizing on them.

We appreciate everyone's support and confidence in our priority directions. It's important that you know
CAO will continue to work hard to provide great value in the year ahead by delivering on these commitments through sustainable operations.

lors que nous fermerons
les livres pour 2007,
je crois que 2008 sera une " grande année! » Je suis heureux d'annoncer que l'ACO a connu une excellente année 2007 dans presque tous les domaines, que ce soit financier, opérationnel ou stratégique, et que nous avons déjà commencé à profiter de cet élan en 2008. Avant de tourner la page, voyons ensemble quelques points saillants de 2007 :

- Organiser le Congrès biennal le plus rentable financièrement de l'histoire de l'ACO. Merci à

Saskatoon et les participants!

- Célébrer le 40 anniversaire de l'arrivée de l'École d'optométrie à Waterloo.

- Un autre Mois de la santé de l'œil réussi. La cohésion nationale pour ce projet est absolument fantastique et fait l'envie de

\section{LETTER TO THE EDITOR}

\section{A "FUNDS-FOR-CHARITY" APPEAL}

The charitable trust, Eye Care India, besides being engaged in correcting errors of refraction by way of spectacles, and cataract blindness by way of $\mathrm{IOL}$ implantation, among poor patients, organises

(i) 3-day Contact Lens Education Program (CLEP) for diploma-optometrists, and

(ii) enlists optometrists in ECl's Optometric Register.

And this is an appeal for your philanthropic readers to donate the sum of US $\$ 200$ each to support ECl's charity work.

To compensate for their contributions in full, one year air-mail complimentary subscription to Optometry Today (India) quarterly journal will be entered in their names ....and the names will be published as donors in the news section of June 2008 issue of OT.

Contributions, in the name of "Optometry Today" (official organ of Eye Care India) may be sent at C4F/216 Janakpuri, New Delhi 110058, India, Phone +91-11-25599839... and must include donor's complete postal address.

This fundraising campaign will be in operation up to 31st May 2008.

Sincerely,

Dr. Naredra Kumar

Trustee, Eye Care India

Editor, Optometry Today

OptometryToday@gmail.com

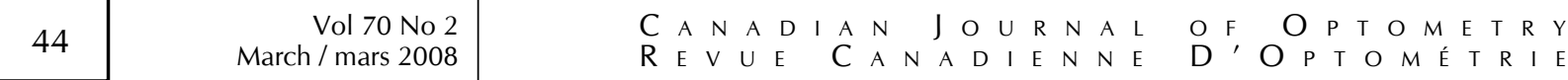




\section{PRESIDENT'S PODIUM MOT DU PRÉSIDENT}

beaucoup d'autres organismes professionnels.

- Mettre en œuvre et surveiller le plan stratégique de l'ACO pour de meilleures relations avec les gouvernements, perfectionner les normes de soins optométriques, améliorer les communications internes et externes et mettre à jour l'infrastructure du bureau de l'ACO. Ce plan directeur comprend également les recommandations du FDO pour améliorer les communications, la formation et la collaboration afin de hausser le profil de l'optométrie.

- Planification provisoire d'une étude d'envergure afin de déterminer la fréquence recommandée des examens de l'œil pour les Canadiens.

- Les rénovations du bureau de l'ACO sont maintenant terminées et ont respecté le budget.

- Embauche d'un nouvel employé qui devient responsable de la surveillance de notre site Web notre futur média d'information, de formation et de communications.

- Contribution financière au forum de l'École d'optométrie de l'Université de Waterloo pour discuter la formation optométrique future.

- Mise sur pied d'un programme d'adhésion gratuite à l'ACO (sur demande) pour tous les étudiants en optométrie inscrits dans l'une ou l'autre des écoles nordaméricaines.

- Réponse au rapport du Bureau de la concurrence.

- Correspondance avec les ministres de la santé provinciaux de la C.-B., du MB et de l'I.-P.-E. sur la nécessité de mettre en œuvre une législation sur les APT.

- Adoption du thème « La communication est la clé »... c'est reconnaître que l'éducation continue et la promotion des services optométriques tant pour le public que pour la profession ouvriront de nombreuses portes qui contribueront à nos succès à venir. 2007 a été une bonne année et nous sommes sur une bonne lancée.

Allons maintenant à notre $8^{\mathrm{e}}$ Forum annuel des leaders optométriques (FDO) de janvier 2008 qui a connu une participation record. C'était notre première réunion nationale de la nouvelle année, qui avait pour but d'examiner, de renouveler et de relancer nos engagements face à nos objectifs pour 2008.

Le FDO 2008 a été une excellente expérience d'échanges ouverts et libres d'idées qui ont donné lieu à un rajeunissement spirituel, qui doit être vécu pour être apprécié. Nous pouvons mieux voir maintenant la forêt et les arbres et je crois que nous continuerons à être gagnants en créant et saisissant les nouvelles possibilités et en capitalisant sur celles-ci.

Nous vous remercions de l'appui et de la confiance que chacun de vous témoigne dans nos orientations prioritaires. Il est important que vous sachiez que l'ACO continuera à travailler d'arrache-pied pour faire de la prochaine année une réussite grâce à des activités durables qu'elle mettra en cuvre pour honorer ses engagements.

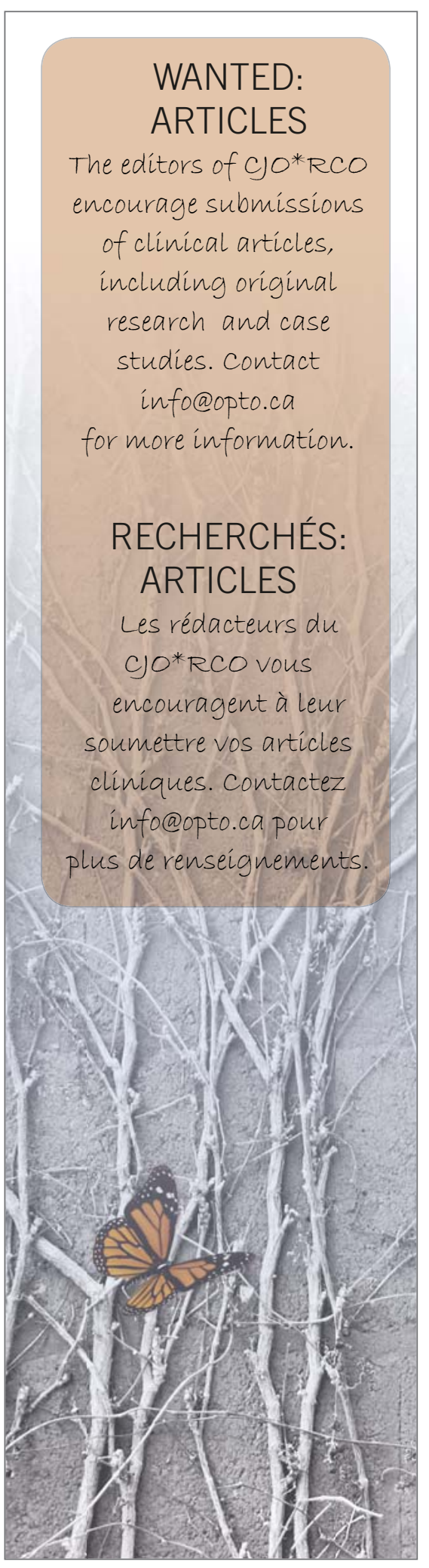

